\title{
EL DESARROLLO DE LAS HABILIDADES DE ARGUMENTACIÓN EN DISCUSIONES LITERARIAS EN SALAS DE CLASES: UNA REVISIÓN DE LA LITERATURA
}

Pelusa Orellana García

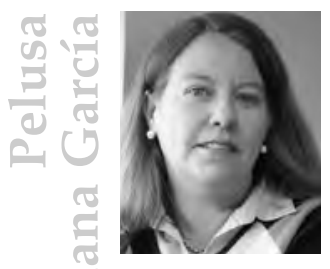

Ph.D. in Education, University of North Carolina at Chapel Hill, U.S.A. Miembro del equipo docente del National Paideia Center. Profesora de Literatura y Metodología del Inglés, Universidad de los Andes, Santiago, Chile. Directora de la Escuela de Pedagogía de la Universidad de los Andes, Santiago, Chile.

Correo electrónico: [porellana@email.unc.edu].

\section{RESUMEN}

El presente artículo señala los avances en la investigación del desarrollo de las habilidades y estrategias de argumentación durante discusiones literarias. La primera parte describe la importancia del desarrollo de estrategias argumentativas y los principales enfoques teóricos utilizados en el estudio de la argumentación. En la segunda parte del artículo, se presenta un análisis crítico de las investigaciones hasta ahora realizadas, en las que se ha explorado el desarrollo y la enseñanza de la argumentación a través de discusiones literarias con alumnos de educación primaria y secundaria. Se describen también los métodos de análisis más utilizados en las investigaciones publicadas y los principales resultados. Finalmente, la tercera parte del artículo expone las líneas de investigación futuras en el campo de la práctica y el ejercicio de la argumentación. 
Palabras clave: argumentación; discusión literaria; contextos dialógicos.

\section{ABSTRACT}

The current article describes recent findings about the development of argumentative skills and strategies in literature discussions. In the first section, I explain the importance of the development of argumentative strategies and the main theoretical approaches on argumentation studies. The second section provides a critical analysis of recent research studies about the development and teaching of argumentation in literature discussions, particularly with elementary and secondary students. I also describe the most commonly used methods of analysis and key findings. Finally, the third section of the article draws on the future areas of research in the fields of argumentative practice.

Key Words: argumentation; literature discussions; dialogic context.

\section{I. ¿POR QUÉ SON IMPORTANTES LAS HABILIDADES DE ARGUMENTACIÓN EN DISCUSIONES LITERARIAS Y DESDE QUÉ PERSPECTIVAS TEÓRICAS HAN SIDO ABORDADAS?}

\section{IMPORTANCIA DE LAS HABILIDADES DE ARGUMENTACIÓN EN DISCUSIONES LITERARIAS}

La argumentación es un proceso dialógico, intelectual y lingüístico mediante el cual los participantes examinan y elaboran aserciones acerca de conceptos, situaciones o ideas. Estas aserciones son fundamentadas, cuestionadas o refutadas por los participantes a medida que analizan y discuten en conjunto. Para ello, los alumnos usan una serie de estrategias intelectuales tanto para 
evaluar la integridad lógica de los argumentos expuestos por otros participantes, como también para elaborar refutaciones y contra argumentos.

La discusión literaria es una de las prácticas metodológicas más usadas en las asignaturas de lenguaje y literatura (Alvermann, O’Brien y Dillon, 1990; Eeds y Wells, 1989). En una discusión literaria, los alumnos analizan ideas y conceptos que un texto sugiere, evaluando los planteamientos del autor a la luz de sus conocimientos y experiencias personales, y de interpretaciones y explicaciones colectivas. A pesar de su empleo frecuente, poco se sabe respecto a la manera en que la discusión literaria constituye un contexto apto para enseñar a los alumnos a generar argumentos lógicos y coherentes.

Un factor que en parte explica la falta de aprovechamiento de esta instancia pedagógica para examinar la argumentación de los alumnos es el hecho de que en muchas salas de clases, la discusión literaria aún conserva un formato en el cual el profesor lidera y ejerce control sobre la participación y el contenido de la discusión (Cazden, 1988; Nystrand, 1997), y es menos frecuente observar discusiones en que la elección de los temas a discutir y la autoridad interpretativa estén realmente en manos de los alumnos (Billings y Fitzgerald, 2002). Alvermann et al. también sostienen que en general los profesores poseen distintas concepciones respecto de lo que es una discusión literaria, y que suele ser frecuente que no exista coherencia entre lo que los profesores definen teóricamente como discusión literaria y la forma en que la aplican en sus asignaturas.

\section{PERSPECTIVAS TEÓRICAS}

El estudio de la argumentación se remonta a los autores de la Grecia y Roma clásicas, siendo probablemente Aristóteles el primero en desarrollar un marco para evaluar la calidad de un argumento desde una perspectiva más bien estructural. En el enfoque aristotélico, el objetivo es distinguir argumentos convincentes de aquéllos 
falaces o bien carentes de lógica. Los enfoques más contemporáneos, sin embargo, han puesto mayor énfasis en aspectos contextuales y sociales que influyen en la creación y desarrollo de un argumento. Por otra parte, la publicación en 1958 del libro de Stephen Toulmin, The Uses of Argument, ha contribuido a generar un mayor interés por examinar la argumentación más allá de los contextos políticos, retóricos o filosóficos en que tradicionalmente se abordaba. En su libro, Toulmin plantea un modelo práctico que identifica argumentos y sus componentes, tanto en textos escritos como en la conversación cotidiana, lo cual ha generado instancias para su investigación en el campo educativo.

Según Toulmin, la argumentación es un proceso en el que no sólo importa saber cuál argumento resulta más convincente, sino que interesa examinar cómo éste se genera y entrelaza en contextos sociales cotidianos. Más que un debate, Toulmin concibe la argumentación como una manera de generar conocimiento y cambios conceptuales a partir de una lectura crítica de la información disponible, tomando en consideración los contextos sociales, históricos y culturales que inciden en la forma de abordar dicha información.

Toulmin plantea que en un argumento pueden identificarse los siguientes seis elementos: 1) la aserción o tesis que un participante expone; 2) la evidencia o bases que sostienen una aseveración; 3) la garantía que sostiene la relevancia de la evidencia; 4) el respaldo, que agrega validez a las garantías; 5) la reserva, que puede ser una objeción, refutación o excepción a una aserción, y 6) el calificativo moral o nivel de certeza que se tiene frente a una aseveración.

Los argumentos de menor calidad se caracterizan por estar conformados por una aserción solamente, o bien una aserción y alguna evidencia de menor peso; mientras que un argumento de mejor calidad puede contener varias aserciones con sus respectivas garantías y evidencias, e idealmente varias reservas que refuten las aserciones originales. Este modelo de argumentación ha sido utilizado en algunas investigaciones acerca de la argumentación generada por los alumnos durante discusiones literarias (Suthers, Toth y Wiener, 1997; 
Pontecorvo y Girardet, 1993; Means y Voss, 1996), discusiones científicas (Osborne, Erduran y Simon, 2004; Simon, Erduran y Osborne, 2006) y matemáticas (Wood, 1999).

Las investigaciones que han estudiado la argumentación en discusiones literarias lo hacen desde perspectivas sociocognitivas y socioculturales. Ambas teorías definen el razonamiento como una habilidad dependiente del contexto social, al tomar de Vygotsky la idea de que el razonamiento individual es una interiorización de procesos originados en la interacción con los demás. Desde el punto de vista sociocognitivo, existe una interacción recíproca entre los aprendices y su entorno; el conocimiento funciona como mediador que orienta las relaciones entre lo que los alumnos saben y lo que no. Kuhn y Udell (2003) explican, por ejemplo, que el desarrollo de habilidades argumentativas depende de la presencia de un ambiente cognitivamente enriquecido (como son las conversaciones grupales en donde los alumnos deben evaluar, construir y responder a argumentos). La perspectiva sociocultural, por otra parte, enfatiza los procesos sociales e históricos que subyacen en el aprendizaje, así como también la mediación semiótica del lenguaje. En este contexto, las discusiones son eventos social y culturalmente situados: la comprensión y el conocimiento se obtienen en forma de «cultura colectiva», como resultado de interacciones verbales entre distintas personas (Bridges, 1979; Almasi, O'Flahavan y Arya, 2001), o lo que Rogoff (1995) define como «apropiación participativa», y Mercer y Littleton (2007) denominan «interpensamiento» (interthinking). Según Vygotsky, la interiorización ocurre cuando las conductas adquiridas mediante la interacción social externa crecen al interior de la mente y se convierten en funciones mentales de orden superior (Bodrova y Leong, 1996).

Una tercera visión teórica está dada por el concepto de dialogismo desarrollado por Mikhail Bakhtin (1981), según el cual el razonamiento es siempre dialógico y en el que la pluralidad de voces a nivel inter e intrapersonal se enriquece por los factores sociales, culturales e ideológicos que los participantes aportan a la discusión (Reznitskaya et al., 2001; Anderson et al., 2001; Clark, Anderson, Kuo, Kim, Archodidou y Nguyen-Jahiel, 2003). Así como la conversación entre 
dos o más personas es una yuxtaposición de voces (Nystrand, 1996), el razonamiento se manifiesta internamente como múltiples voces que dialogan en la mente de una persona para construir significado. Tomasello (1999) explica esto en los siguientes términos:

El uso de símbolos lingüísticos en la interacción discursiva en la que se contrastan y comparten explícitamente distintas perspectivas, otorgan el material básico a partir del cual los niños de todas las culturas construyen las representaciones cognitivas flexibles y de múltiples perspectivas -quizá incluso dialógicas- que otorgan al conocimiento humano gran parte de su asombroso y único poder (p. 163).

\section{II. ¿QUÉ SE SABE ACERCA DEL DESARROLLO DE HABILIDADES DE ARGUMENTACIÓN EN DISCUSIONES LITERARIAS; QUÉ MÉTODOS ANALÍTICOS SE HAN USADO Y CUÁLES HAN SIDO LAS CONCLUSIONES MÁS SIGNIFICATIVAS?}

\section{LO QUE SE SABE ACERCA DEL DESARROLLO DE HABILIDADES DE ARGUMENTACIÓN}

El interés por estudiar el desarrollo de la argumentación en discusiones a nivel escolar es bastante reciente (van Eemeren, Grootendorst y Schnoeck, 1996). A comienzos de este siglo, la mayoría de las publicaciones al respecto sólo se centraba en aspectos teóricos y había pocos estudios empíricos en los que se observara a alumnos discutiendo textos literarios, o conceptos aprendidos en las distintas disciplinas (Kuhn y Udell, 2003). Este mayor interés por el desarrollo de la argumentación en la sala de clases radica en la creciente importancia de la interacción social en el pensamiento individual (Reznitskaya et al., 2001; Cazden, 1988; Commeyras, 1994; Kuhn, 1992), y del hecho que la argumentación constituye una actividad inherente al ser humano (Voss y Van Dyke, 2001; Wertsch, 1981). 
Desde la perspectiva de la psicología cognitiva, el estudio de la argumentación en discusiones de sala de clase otorga un lente mediante el cual ha podido observarse la manera en que los niños construyen argumentos en conjunto: cómo el desarrollo de habilidades de argumentación va más allá de la solución de problemas (i.e., Kuhn, 1991; Kuhn y Udell, 2003; Anderson et al., 1997), y qué factores inhiben o facilitan la argumentación de calidad. Los estudios han sugerido que las competencias argumentativas se pueden analizar en niños a partir de los tres años de edad (Kuhn y Udell, 2003; Anderson et al., 1997; Orsolini, 1993; Stein y Miller, 1993; Stein y Albro, 2001). Anderson et al. (1997) también han advertido que los niños pequeños suelen producir argumentos en los que existen saltos lógicos y suelen no tener conclusiones explícitas, pero que sin embargo constituyen argumentos tanto desde una perspectiva estructural como desde el punto de vista del contenido. Pese a la ausencia muchas veces de referencias y conclusiones lógicas, los argumentos infantiles parecen tener cierta coherencia porque el contexto en el que se desarrollan facilita la clarificación de posibles ambigüedades. Esta observación es consistente con lo que Schlessinger, Keren-Portnoy y Paruscht (2001) plantean en el sentido de que los argumentos contienen una estructura subyacente o línea de razonamiento en la cual algunos pasos, si bien no se enuncian verbalmente, pueden entreverse cuando la estructura del discurso se evidencia.

Las investigaciones también sostienen que la adquisición de habilidades de argumentación depende del desarrollo evolutivo (Felton y Kuhn, 2001), que su proceso es distinto entre hombres y mujeres, y que existen factores culturales que influyen en la calidad del discurso argumentativo (Dawe, 1934; Kuhn, 1991). Por ejemplo, los alumnos con mayor desarrollo de habilidades de orden superior y que poseen mayor capital cultural, generan argumentos de mayor calidad (Kuhn, 1992; Means y Voss, 1996; Perkins, 1985; Voss, Blais, Means, Greene y Ahwesh, 1986). Osborne et al. (2004) sostienen que las habilidades argumentativas se adquieren sólo si son enseñadas de manera explicita, y si son empleadas frecuentemente por los profesores como un modelo de razonamiento (Kuhn y Udell, 2003). 
En investigaciones más recientes se ha examinado el grado de transferencia de las habilidades de argumentación entre distintos dominios cognitivos (Reznitskaya, Anderson y Kuo, 2007), como por ejemplo: de qué manera los alumnos son capaces de aplicar las habilidades argumentativas que emplean en discusiones literarias al redactar textos argumentativos. Algunas investigaciones (e.g. Reznitskaya et al., 2007; Kuhn, Shaw y Felton, 1997) concluyen que la participación en discusiones literarias incide positivamente en la producción de argumentos escritos, aunque no está claro que un mejor discurso argumentativo escrito se deba exclusivamente a la participación en discusiones grupales.

Otro aspecto interesante que ha sido observado en conversaciones y discusiones literarias entre alumnos de educación primaria es la apropiación de estrategias de argumentación por parte de los alumnos que, careciendo de estas estrategias, son capaces de incorporarlas a su repertorio cuando ven que otros alumnos las utilizan de manera exitosa para generar o rebatir un argumento (Anderson et al., 2001; Reznitskaya et al., 2007).

\section{MÉTODOS ANALÍTICOS}

Hasta el año 2000 existían pocos estudios empíricos sobre el desarrollo de la argumentación en las discusiones de clase. El incremento en los estudios a partir de esa fecha ha incidido también en una gran variedad de enfoques metodológicos para examinar aspectos tales como el uso de estrategias argumentativas, la calidad de los argumentos, y su transferencia a otros dominios cognitivos. De manera similar, este aumento en el interés por el tema ha generado también una gran diversidad en lo que respecta a las poblaciones estudiadas. Las investigaciones publicadas incluyen muestras provenientes de todo tipo de escuelas (públicas y privadas, urbanas y rurales, elementales y superiores) reflejando un mayor espectro sociocultural y étnico, así como también variados niveles de escolaridad. El tamaño de las muestras varía entre 20 y varios cientos de alumnos participantes, aspecto que también determina el grado de generalización de las conclusiones obtenidas (ver, por 
ejemplo, Reznitskaya et al., 2001; Reznitskaya et al., 2007; Anderson et al., 2001; Chinn et al., 2002). Hay gran cantidad de estudios centrados en la educación básica, en particular entre segundo y cuarto grado, así como también estudios comparativos en que se analiza el empleo de estrategias argumentativas en muestras de adolescentes y adultos (Kuhn, Ho y Adams, 1979; Kuhn y Udell, 2003).

Respecto a los contextos de discusión, los investigadores han observado discusiones con mayor o menor grado de estructuración. Un contexto bastante estudiado es el de la metodología denominada «razonamiento colaborativo» (collaborative reasoning) (Chinn et al., 2002; Anderson, Chinn, Waggoner y Nguyen-Jahiel, 1998; Chinn et al., 1998; Waggoner et al., 1995), y existen muy pocas investigaciones en las que se ha analizado el razonamiento y la argumentación en discusiones en parejas y / o grupos pequeños (Kuhn y Udell, 2003; Pontecorvo y Girardet, 1993), círculos literarios y seminarios socráticos (Orellana, 2008).

En su mayoría, los métodos usados son la observación apoyada de videograbación y / o grabación de audio para registrar la presencia de argumentos en las conversaciones de los alumnos. Una vez grabadas, las conversaciones son transcritas mediante un procesador de texto, para luego codificarse con el fin de identificar argumentos y sus componentes. Una vez codificados los argumentos y componentes es posible realizar distintos análisis cuantitativos y cualitativos sobre presencia y calidad de argumentos, así como también la estructura de los mismos, el aprovechamiento de estrategias específicas y de redes argumentativas (Chinn y Anderson, 1998). En estudios en los que se ha querido examinar la transferencia de las habilidades argumentativas a otros dominios, como por ejemplo la escritura o distintas asignaturas, los investigadores incluyen cuestionarios aplicados antes y después de las discusiones, o bien han recopilado ensayos escritos antes y después. Otra técnica usada en investigaciones es el recuerdo de la estructura argumentativa (cf. Chambliss y Murphy, 2002) para después determinar la replicación de ciertas estructuras en el discurso oral o escrito de los alumnos. 


\section{CONCLUSIONES}

Si bien existen varios estudios científicos en los que se han examinado algunos aspectos del empleo de las discusiones literarias, hay muy pocas investigaciones en las que se aborde -en concretosu relación con el desarrollo de estrategias argumentativas en los alumnos. A pesar de ello, pueden desprenderse algunas conclusiones importantes acerca del desarrollo de estrategias argumentativas, en particular cuando los alumnos realizan discusiones literarias.

En primer lugar, los estudios han demostrado que la argumentación es un fenómeno observable, incluso en niños pequeños. El desarrollo y la calidad del discurso argumentativo dependen de una serie de factores tanto evolutivos como socioculturales y cognitivos. Aun así, se ha comprobado que los alumnos logran adquirir estas estrategias cuando las aprenden formalmente, en un contexto significativo (por ejemplo, una discusión literaria en la cual inciden factores tales como puntos de vista más o menos subjetivos, conocimiento previo, capital cultural, ambigüedad y grado de dificultad del texto, motivación, etcétera). Para alcanzar un mejor dominio del discurso argumentativo, los alumnos se beneficiarían aún más si el profesor exhibe estas estrategias y sirve de modelo a seguir.

En segundo lugar, las investigaciones han revelado que el uso de estrategias argumentativas es acumulativo e imitativo; es decir, al observar el empleo de las mismas, los alumnos las incorporan a su repertorio de estrategias y adquieren mejor desempeño en su empleo. Las investigaciones han demostrado que los alumnos también generan más y mejores argumentos cuando deben aplicar estas estrategias con mayor frecuencia. Por tanto, cuando los maestros utilizan las discusiones literarias como una herramienta que apunte al uso de habilidades de orden superior, será más fácil identificar y examinar la producción argumentativa de los alumnos.

Respecto a los métodos de análisis de la argumentación, existen varias dificultades en la manera de evaluar la calidad del discurso argumentativo, aspecto que impide en gran medida generalizar las 
conclusiones, por lo que muchas investigaciones mantienen aún un carácter exploratorio. También se hace necesario analizar de qué manera la estructura del discurso facilita o inhibe la producción de argumentos, determinar qué tipo de estructuras discursivas se relacionan con argumentos de mejor calidad, de qué manera se conjugan aspectos tales como estructuras de autoridad y dinámicas de grupo, y aspectos culturales con la producción argumentativa. A pesar de esto, las conclusiones obtenidas a partir de estudios empíricos permiten establecer un marco común que sirve de base para ir generando nuevo conocimiento sobre cómo abordar aspectos tales como la calidad y estructura de los argumentos y los tipos de discusiones que los fomentan.

\section{FUTURAS LÍNEAS DE INVESTIGACIÓN}

El interés creciente por examinar lo que ocurre cuando los alumnos discuten obras literarias generará, sin duda, un amplio espectro de estudios de investigación orientados a comprender los procesos cognitivos que se desarrollan en dicho contexto. Al respecto, existen muchos y diversos aspectos que podrían examinarse y entregar conclusiones que contribuyan a aprovechar al máximo estas instancias de aprendizaje. A continuación enunciaremos algunos que parecerían de interés, sin perjuicio de que pudieran agregarse muchísimas otras condiciones y fenómenos propios de las discusiones literarias.

Una primera línea de investigación consiste en examinar el desarrollo de la argumentación en diferentes contextos discursivos, más allá de la tradicional discusión grupal. Si bien existen varias investigaciones sobre las prácticas argumentativas de los alumnos en discusiones literarias, no se han analizado discusiones menos tradicionales, como son, por ejemplo, las discusiones virtuales a través de sitios web o chat rooms (Morgan y Beaumont, 2003), los seminarios socráticos (Adler, 1988; Billings y Fitzgerald, 2002; Orellana, 2008), los clubes de lectura y los círculos literarios (Sandora, Beck y McKeown, 1999). Un análisis comparativo de lo que ocurre con la argumentación en el marco de distintos formatos discursivos puede 
proporcionar una visión más global sobre cómo fortalecer las estrategias de argumentación de los alumnos, enfatizando aquellos formatos que mejor fomentan estas estrategias. De la misma manera, puede resultar interesante determinar el rol que cumple el tipo de texto literario que se discute en la generación de más argumentación y en la calidad de la misma. Se ha observado, por ejemplo, que cuando las discusiones giran en torno a textos más filosóficos, se generan más argumentos y de mejor calidad que cuando se emplean textos menos complejos o menos ambiguos (Orellana, 2008).

Una de las principales interrogantes respecto al estudio de la argumentación es la evaluación de los argumentos y la subjetividad que puede interferir en dicho proceso. Al respecto, la investigación acerca de distintas formas de evaluar la calidad del discurso argumentativo es un tema interesante de analizar y desarrollar, dado que no existen estudios que comparen distintos métodos. En este sentido también resulta importante abordar de manera comparativa distintos modelos, marcos analíticos, y epistemologías con las que se mire la argumentación al interior de las discusiones literarias (cf. Clark, Sampson, Weinberger y Erkens, 2007).

Finalmente, hay una serie de aspectos de carácter práctico que podrían examinarse con el fin de perfeccionar el uso de las discusiones literarias para el desarrollo de habilidades argumentativas. Se sabe, por ejemplo, que la argumentación debe ser enseñada formal y conscientemente a los alumnos, y que éstos deben observar estrategias concretas antes de incorporarlas a su repertorio. Desde este punto de vista, interesa analizar cuáles son las habilidades argumentativas que el profesor debe demostrar a sus alumnos y cuál es la mejor manera de hacerlo. También resulta importante determinar si existen herramientas con las cuales identificar, por un lado, el buen empleo de estrategias argumentativas, y por otro, la manera de fomentar su aplicación en clases. Si los profesores conocen estas estrategias, las usan y modelan con frecuencia en sus clases, y las ejercitan con sus alumnos, mejorará la calidad y aumentará la cantidad de argumentos que los alumnos construyan cuando discutan textos literarios o se involucren en cualquier 
discusión grupal. La incorporación de estas estrategias ha de ser paulatina y creciente, entregando a los alumnos el andamiaje necesario para que poco a poco se atrevan a argumentar constructivamente, usando el pensamiento crítico de manera coherente. Para ello hace falta generar conocimiento que pueda ser aplicable por todos los profesores y en todos los contextos educativos. Sólo así lograremos que, en las escuelas, todos los alumnos adquieran las habilidades de orden superior cada vez más necesarias para resolver los desafíos de una sociedad basada en el conocimiento.

\section{BIBLIOGRAFÍA}

ADLER, M. (1998), The Paideia proposal, Touchstone Books, New York.

ALMASI, J.; O’FLAHAVAN, J. \& ARYA, P., «A comparative analysis of student and teacher development in more and less proficient discussions of literature», Reading Research Quarterly, 36 (2), 2001, p. 96-120.

ALVERMANN, D.; O’BRIEN, D. \& DILLON, D., «What teachers do when they say they're having discussions of content area reading assignments? A qualitative analysis», Reading Research Quarterly, 25, no. 4, 1990, p. 296-322.

ANDERSON, R.C.; CHINN, C.; WAGGONER, M.A. \& NGUYEN, K., «Intellectually stimulating story discussions», in OSBORN, J. \& LEHR, F. (eds.) Literacy for all: issues in teaching and learning, Guilford, New York, 1998, p. 170-199.

ANDERSON, R.; NGUYEN-JAHIEL, K.; MCNURLEN, B.; ARCHODIDOU, A.; KIM, S.; REZNITSKAYA, A.; TILLIMANNS, M. \& GILBERT, L., «The snowball phenomenon: Spread of ways of talking and ways of thinking across groups of children», Cognition and Instruction, 19 (1), 2001, p. 1-46. 
BAKHTIN, M., The dialogic imagination: four essays, University of Texas Press, Austin, Texas, 1981.

BILLINGS, L. \& FITZGERALD, J., «Dialogic Discussion and the Paideia Seminar», American Educational Research Journal, 2002, p. 907-941.

BODROVA, E. \& LEONG, D., Tools of the Mind: The Vygotskyan approach to early childhood education, Englewood Cliffs, Merrill, NJ, 1995.

BRIDGES, D., Education, democracy, and discussion, National Foundation for Educational Research, Windsor, England, 1979.

CAZDEN, C.B., Classroom discourse: The language of teaching and learning, Heinemann, Portsmouth, NH, 1988.

CHAMBLISS, M. \& MURPHY, K., «Fourth and fifth graders representing the argument structure in written texts», Discourse Processes, 34 (1), 2002, p. 91-115.

CHINN, C.; ANDERSON, R.C. \& WAGGONER, M., «Patterns of discourse during two kinds of literature discussion», Reading Research Quarterly, 36, 2001, p. 378-411.

CHINN, C.A. \& ANDERSON, R.C., «The structure of discussions that promote reasoning», Teachers College Record, 100, 1998, p. 315-368.

CLARK, A.; ANDERSON, R.C.; KUO, L.; KIM, I.; ARCHODIDOU, A. \& NGUYEN-JALIEL, K., «Collaborative reasoning. Expanding ways for children to talk and think in school», Educational Psychology Review, 15, 2003, p. 181-198.

COMMEYRAS, M., «Promoting critical thinking through dialogical-thinking reading lessons», The Reading Teacher, 46, 1993, p. 486-494.

DAWE, H.C., «An analysis of 200 quarrels of preschool children», Child Development, 5, 1934, p. 139-157. 
EEDS, M. \& WELLS, D., «Grand Conversations: an exploration of meaning construction in literature study groups», Research in the teaching of English, 23, 1989, p. 4-29.

FELTON, M. \& KUHN, D., «The development of argumentative discourse skills», Discourse Processes, 32, 2001, p. 135-153.

KUHN, D., The skills of argument, Cambridge University Press, Cambridge, 1991.

KUHN, D.; SHAW, V. \& FELTON, M., «Effects of dyadic interaction on argumentative reasoning», Cognition and Instruction, 15(3), 1997, p. 287-316.

KUHN, D. \& UDELL, W., «The Skills of Argument», Child Development, 74 (5), 2003, p. 1245-1260.

KUHN, D.; HO, V. \& ADAMS, C., «Formal reasoning among preand late adolescents», Child Development, 50, 1979, p. 1128-1135.

MEANS, M. \& VOSS, J., «Who reasons well? Two studies of informal reasoning among children of different grade, ability, and knowledge levels», Cognition and Instruction, 14 (2), 1996, p. 139-178.

MERCER, N. \& LITTLETON, K., Dialogue and the Development of Children's Thinking: a sociocultural approach, Routledge, New York, 2007.

MORGAN, W. \& BEAUMONT, G., "A dialogic approach to argumentation: using a chat room to develop early adolescent students' argumentative writing», Journal of Adolescent and Adult Literacy, 47:2, 2003, p. 146-167.

NYSTRAND, M., Opening Dialogue. Understanding the dynamics of language and learning in the English classroom, Teachers College Press, New York, 1996.

NYSTRAND, M.; GAMORAN, A.; KACHUR, R. \& PENDERGAST, $C .$, Opening Dialogue.Understanding the dynamics of language and learning in the English classroom, Teachers College Press, New York, 1997. 
ORELLANA, P., «Maieutic frame presence and degree of quantity and quality of argumentation», in A Paideia Seminar, Unpublished doctoral dissertation, The University of North Carolina at Chapel Hill, 2008.

ORSOLINI, M., «Dwarfs do not shoot: An analysis of children's justifications», Cognition and Instruction, 11, 1993, p. 281-97.

OSBORNE, J.; ERDURAN, S. \& SIMON, S., «Enhancing the quality of argumentation in science classrooms», Journal of Research in Science Teaching, 4 (10), 2004, p. 994-1020.

PERKINS, D., «Post-primary education has little impact upon informal reasoning», Journal of Educational Psychology, 77, 1985, p. $563-571$.

PONTECORVO, C. \& GIRARDET, H., «Arguing and reasoning in understanding historical topics», Cognition and Instruction, 11, 1993, p. 365-395.

REZNITSKAYA, A.; ANDERSON, R.C.; MCNURLEN, B.; NGUYEN-JAHIEL, K.; ARCHODIDOU, A. \& KIM, S.Y., «Influence of oral discussion on written argument», Discourse Processes, 32, 2001, p. 155-175.

REZNITSKAYA, A.; ANDERSON, R. \& KUO, L., «Teaching and learning argumentation», The Elementary School Journal, 107 (5), 2007, p. 450-472.

ROGOFF, B., «Observing sociocultural activity on three planes: Paricipatory appropriation, guided participation, and apprenticeship», in WERTSCH, J.B.; DEL RIO, P. \& Alvarez, A. (eds.), Sociocultural Studies of Man, Cambridge University Press, Cambridge, England, 1995, p. 139-164.

SANDORA, C.; BECK, I. \& MCKEOWN, M., «A comparison of two discussion strategies on students' comprehension and interpretation of complex literature», Reading Psychology, 20, 1999, p. 177-212, 70-103. 
STEIN, N. \& ALBRO, E., «The Origins and Nature of Arguments: Studies in Conflict Understanding, Emotion, and Negotiation», Discourse Processes, 32, 2, 2001, p. 113-133.

STEIN, N.L. \& MILLER, C.A., «The development of memory and reasoning skill in argumentative contexts: evaluating, explaning, and generating evidence» in GLASER, R. (ed.), Advances in instructional psychology, Erlbaum, Hillsdale N.J., 1993, p. 285-335.

SIMON, S.; ERDURAN, S. \& OSBORNE, J., «Learning to teach argumentation: Research and development in the science classroom», International Journal of Science Education, 28 (2-3), 2006, p. $245-260$.

SUTHERS, J.; TOTH, E. \& WEINER, A., «An Integrated Approach to Implementing Collaborative Inquiry in the Classroom», Proceedings of Computer Supported Collaborative Learning (CSCL'97), December 10-14, Toronto, 1997, p. 272-279.

TOMASELLO, M., The Cultural Origins of Human Cognition, Harvard University Press, 1999.

TOULMIN, S., The Uses of Argument, Cambridge University Press, London, U.K., 1958.

VAN EEMEREN, F.H.; HOUTLOSSENT \& SNOECK, F., Argumentative Indicators in Discourse: A Pragma Dialectical Study, Springer, Dordrecht, The Netherlands, 2003.

VOSS, J.F.; BLAIS, J.; MEANS, M.L.; GREENE, T.R. \& AHWESH, E., «Informal reasoning and subject matter knowledge in the solving of economics problems by naive and novice individuals», Cognition and Instruction, 3, 1986, p. 269-302.

VOSS, J.F. \& VAN DYKE, J.A., «Argumentation in psychology: Background comments», Discourse Processes, 32, 2001, p. 89-111.

WAGGONER, M.A.; CHINN, C.A.; ANDERSON, R.C. \& YI, H., «Collaborative reasoning about stories», Language Arts, 72, 1995, p. 582-589. 
WERSCH, J., Voices of the mind: a sociocultural approach to mediated action, Harvard University Press, Cambridge, MA, 1995.

WOOD, T., «Creating a Context for Argument in Mathematics Class», Journal for Research in Mathematics Education, Vol. 30, No. 2, 1999, p. 171-191. 